\title{
Imaging in Diabetic Foot
}

\section{Sunita Dhanda* and Swee Tian Quek}

Department of Diagnostic Imaging, National University Hospital, Singapore

*Corresponding author: Dr. Sunita Dhanda, Department of Diagnostic Imaging, National University Hospital, 5 Lower Kent Ridge Road, 119074, Singapore, Tel: +65-90619157; E-mail: sunitadhanda63@gmail.com

Received date: November 03, 2017; Accepted date: November 04, 2017; Published date: November 08, 2017

Copyright: ( 2017 Dhanda S, et al. This is an open-access article distributed under the terms of the Creative Commons Attribution License, which permits unrestricted use, distribution, and reproduction in any medium, provided the original author and source are credited.

\section{Imaging in Diabetic Foot}

Foot complications in diabetes need early diagnosis to start timely therapy and prevent amputation. Diabetic foot remains a challenge for the clinicians due to confusing clinical picture and associated complications. It may present as neuroarthropathy, septic arthritis, osteomyelitis, ischemic devitalised bone or as soft tissue complications such as cellulitis, myositis, ulceration, callus formation, sinus tracts, abscess, muscle denervation, tenosynovitis etc. Awareness of the various imaging findings of a diabetic foot, their relevance to the therapeutic decisions and correct usage of various imaging modalities to answer pertinent clinical questions are very important to improve the patient management and to reduce complications and morbidity [1].

Plain radiography is the preferred first line imaging investigation (Figure 1).



Figure 1: Advanced neuroarthropathy (Charcot's foot): AP radiograph of the left foot (a) demonstrates disorganization and lateral subluxation of the 1st to 5th tarsometatarsal joints (Lisfranc's dislocation) with increased osseous density and some degree of fusion across the joints (black arrows). Old fractures of 3rd to 5th metatarsals are also noted (white arrows). Axial fat suppressed (FS) T2 weighted (W) MR image (b) confirms the findings of joint destruction (curved arrows) however demonstrates lack of bone marrow edema or joint effusion indicative of chronic neuroarthropathic changes without features of osteomyelitis or septic arthritis. (Reproduced from EPOS ECR 2016, DOI: http:// dx.doi.org/10.1594/ecr2016/C-1086)

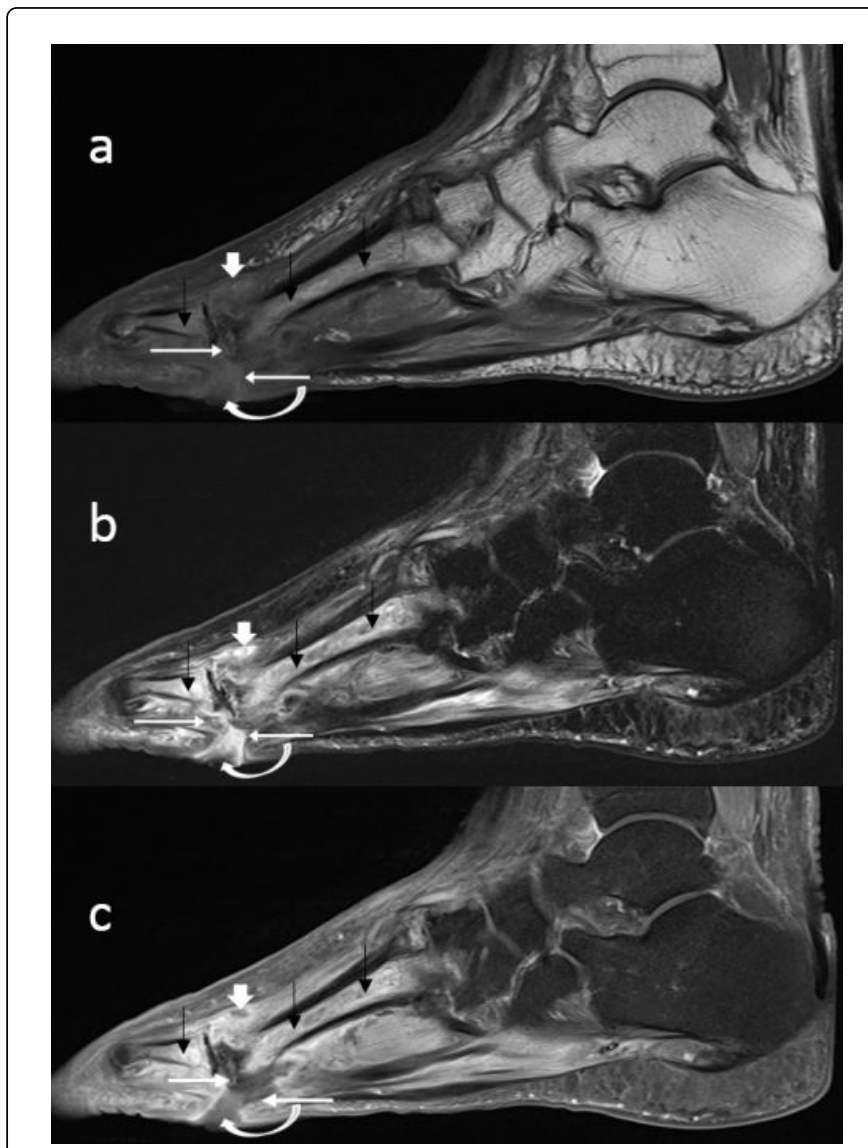

Figure 2: Septic arthritis: Patient with uncontrolled diabetes presented with forefoot ulceration with pain. Sagittal T1W (a), FS T2W (b) and post gadolinium FS T1W (c) images show an irregular ulcer in the plantar skin at the level of the 2nd metatarsal head (curved white arrow). There is subluxation of the adjoining 2nd metatarsophalangeal joint with articular erosions and a fluid collection within the joint, communicating with the skin ulcer ventrally (thin white arrows) and extending into the soft tissue over 2nd metatarsal head dorsally (thick white arrow) suggestive of septic arthritis. Altered marrow signal, appearing hypointense on $\mathrm{T} 1 \mathrm{~W}$, hyperintense on $\mathrm{T} 2 \mathrm{~W}$ image and showing prominent contrast enhancement is seen involving the majority of the 2nd metatarsal and proximal phalanx (thin black arrows) suggestive of osteomyelitis. Surrounding muscles, fascial planes and subcutaneous fat are edematous. (Reproduced from EPOS ECR 2016, DOI: http://dx.doi.org/10.1594/ecr2016/C-1086) 
Page 2 of 3

It can show osseous structures and joint spaces well however it is neither sensitive nor specific. The detection rate and accuracy is low, especially in the scenario of early infection or neuroarthropathy, due to its lack of adequate demonstration of the soft tissues [2]. In addition, Charcot's foot and osteomyelitis may show overlapping radiographic features [2]. Triple phase bone scintigraphy is sensitive however lacks anatomic details and specificity. Hence, often it is used as a screening test or complimentary to labelled leukocyte scintigraphy $[3,4]$.

MRI scanning is the gold standard for differentiating the various pathologies in a diabetic foot [2]. As foot infections in diabetic patients tend to be intractable, early introduction of therapy is paramount to prevent progression to gangrene and consequent amputation. MRI can detect early bone and soft tissue abnormalities with resultant early therapeutic intervention. MRI with its excellent contrast resolution and anatomic detail is an essential tool to help differentiate osteomyelitis from neuropathic arthropathy, identify conditions requiring surgical interventions from those that can be managed conservatively and map the extent of infection. It can thus help limit the area of resection. Septic arthritis commonly involves joints that are adjacent to a callus, ulcer or sinus tract (Figure 2).

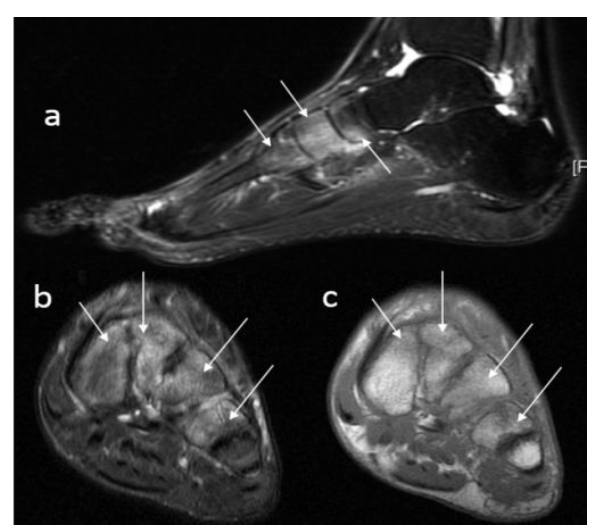

Figure 3: Early neuroarthropathy affecting the bones of the mid foot without frank articular erosion, joint destruction or disorganisation. Marrow edema (thin arrows) is seen in the midfoot as a hyperintense signal on sagittal STIR (a) and coronal FS T2W (b) images and hypointense signal on coronal T1W image (c). No joint effusion, cellulitis, callus, ulcer or sinus tract is seen adjacent to the affected bones. Incidental note is made of ankle joint effusion. (Reproduced from EPOS ECR 2016, DOI: http://dx.doi.org/ 10.1594/ecr2016/C-1086)

There are associated soft tissue changes such as skin cellulitis, abscesses, myositis and tenosynovitis. Subchondral marrow shows T1 hypointense and T2/STIR hyperintense signal intensity on MRI. In contrast, neuroarthropathy is not related to overlying skin or soft tissue changes and commonly involves multiple midfoot bones (Figure 1) $[2,5]$. MRI shows marrow changes in the periarticular and subchondral distribution. Acute neuroarthropathy may mimic osteomyelitis with T1 hypointense and T2/STIR hyperintense signal intensity and may require other secondary signs for differentiation (Figure 3).

"Ghost sign" on MRI has been shown to be a distinguishing feature between acute neuroarthropathy and neuroarthropathy with a superimposed infection. Ghost sign is described as "disappearance" of the bones on T1-weighted images and "reappearance" on T2-weighted images or contrast enhanced images and is indicative of a superimposed osteomyelitis (Figure 1). The ghost sign is absent in the uninfected neuroarthropathic foot. Chronic neuroarthropathy typically shows either normal or low signal in the marrow on both $\mathrm{T} 1$ and T2 weighted images. Features include joint instability, dislocation, destruction, disorganization, increased bone density, joint debris and deformity. Presence of subchondral cysts and intraarticular bodies with absence of the secondary signs for osteomyelitis support neuroarthropathy without infection (Figure 4) $[1,2,5]$. However, this distinction may sometimes be difficult based on MRI alone. Labelled leukocyte scintigraphy is complementary to MRI in making this diagnosis and is a useful tool for follow up of the patients with osteomyelitis (Figure 4) [4].



Figure 4: Advanced neuroarthropathy evaluated with MRI, bone scan and labelled leukocyte scintigraphy. Sagittal T1W (a), FS T2W (b) and post gadolinium FS T1W (c) images show flattening with marked articular irregularity along both aspects of the ankle mortise and talonavicular joint with loss of articular cartilage and joint space (straight white arrows). Subtalar joint is disorganized with subluxation and collapse of joint space (curved white arrow). Focal subchondral cystic changes are seen along the ankle mortise. No significant joint effusion is noted. Paucity of joint effusion, lack of overlying soft tissue swelling and focal asymmetric pattern of subchondral changes favour neuropathic arthropathy over septic arthritis. Coronal T1W (d) and FS T2W (e) images show a large skin ulceration overlying the lateral malleolus with adjoining cellulitis. Bone scan (f,g) shows positive triple phase uptake at the left talotibial joint and the talus. Bony infection could not be excluded based on the bone scan. Small focal uptake is seen in the lateral malleolar region, suspicious for bony infection. Labelled leukocyte scintigraphy done to assess for any infection shows a superficial curvilinear increased radiotracer activity in the region of left distal lateral malleolus in the blood pool phase (h). This focal uptake appears circumferential on the delayed 4hour phase (i) suggesting soft tissue localization. The concerned left tibio-talar and subtalar joints do not show significant increased labelled leukocyte accumulation to suggest infection.

In summary, early detection of foot changes in diabetic patients, especially differentiation of neuropathic changes from early infection 
Page 3 of 3

and mapping the extent of disease are paramount to start timely treatment and reduce long term morbidity. Hence, imaging plays a significant role in management of diabetic foot.

\section{References}

1. Paik E, Wissman RD (2005) Distinguishing Imaging Characteristics of the Diabetic Foot. Infect Dis Clin Pract 13: 303-305.

2. Cunli Y, Ankit T (2013) A Pictorial Review of Diabetic foot Manifestations. Med J Malaysia 68: 279-289.
3. Jay PR, Michelson JD, Mizel MS, Magid D, Le T (1999) Efficacy of threephase bone scans in evaluating diabetic foot ulcers. Foot Ankle Int 20: 347-355.

4. Palestro CJ, Love C (2009) Nuclear medicine and diabetic foot infections. Semin Nucl Med 39: 52-65.

5. Donovan D, Schweitzer ME (2010) Use of MR Imaging in Diagnosing Diabetes-related Pedal Osteomyelitis. RadioGraphics 30: 723-736. 